\title{
Effect of Combined Statin and Antihypertensive Therapy in Patients with Hypertension: A Systematic Review and Meta-Analysis
}

\author{
Ying Wang $^{\mathrm{a}, \mathrm{b}} \quad$ Long Jiang $^{\mathrm{c}} \quad$ Shu-Jun Feng ${ }^{\mathrm{d}} \quad$ Xin-Ying Tang $^{\mathrm{e}}$ Ze-Min Kuang ${ }^{\mathrm{b}}$ \\ a The Second Affiliated Hospital of Henan University of Science and Technology, Luoyang, China; ${ }^{b}$ Department of \\ Hypertension, Beijing Anzhen Hospital of Capital Medical University, Beijing, China; ' Department of Cardiology, \\ The Second Affiliated Hospital of Nanchang University, Nanchang, China; ${ }^{d}$ Hunan Province Key Laboratory of \\ Tumor Cellular and Molecular Pathology, Cancer Research Institute, Hengyang School of Medicine, University of

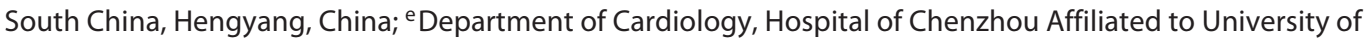 \\ South China, Chenzhou, China
}

\section{Keywords}

Antihypertensive therapy - Cardiovascular outcomes .

Hypertension · Meta-analysis · Statin

\begin{abstract}
Introduction: This meta-analysis aimed to explore the preventive effects of combined statin and antihypertensive therapy on major cardiovascular outcomes in patients with hypertension. Methods: PubMed, Embase, and the Cochrane Library databases and reference lists of published studies were systematically searched throughout October 9, 2019. Studies designed as randomized controlled trials and investigating the effects of combined statin and antihypertensive therapy versus antihypertensive therapy alone were included. Data abstraction and quality of included studies were assessed by 2 independent authors. The summary results were calculated using relative risks (RRs) with $95 \%$ Cls employing a random-effects model. Results: A total of 8 randomized controlled trials including 38,618 patients were finally enrolled. The summary RRs indicated that the combined therapy significantly reduced the risk of major adverse cardiovascular events compared with antihypertensive therapy alone
\end{abstract}

(RR 0.79; 95\% Cl 0.71-0.88; $p<0.001$ ). Furthermore, the patients in the combined therapy group also experienced less myocardial infarction (RR $0.67 ; 95 \% \mathrm{Cl} 0.53-0.84 ; p=0.001$ ) and stroke risks (RR 0.82; $95 \% \mathrm{Cl} 0.72-0.94 ; p=0.005$ ), while no significant difference was observed between combined therapy and antihypertensive therapy alone regarding cardiac death (RR 0.96; 95\% $\mathrm{Cl} 0.84-1.08 ; p=0.465$ ) and all-cause mortality (RR $0.95 ; 95 \% \mathrm{Cl} 0.86-1.04 ; p=0.277$ ). Conclusion: These findings suggested that combined statin and antihypertensive therapy was associated with more cardiovascular benefits compared with antihypertensive therapy alone.

(c) 2020 S. Karger AG, Basel

\section{Introduction}

High blood pressure is more frequently observed in individuals aged more than 25 years, affecting more than $40 \%$ of adults worldwide. It is the leading cause of death or disability [1-3]. The American Society of Hypertension collaboration group in 2009 defined hypertension as a progressive vascular syndrome caused by a series of complex and intervening causes evidenced by an increase

$\begin{aligned} & \text { karger@karger.com } \\ & \text { www.karger.com/crd }\end{aligned}$
Karger ${ }^{\prime /}$


in blood pressure [4]. Furthermore, hypertension is considered as the most important risk factor for endovascular atherosclerosis and induces greater atherosclerotic cardiovascular disease (CVD) risks when combined with other cardiovascular risk factors $[5,6]$. However, the residual risk of cardiovascular outcomes remains high owing to patients having various cardiovascular risk factors. Therefore, combined statin and antihypertensive therapy, even polypills, should be used according to risk-based approaches, and the treatment with combined strategies should be based on the absolute risk of cardiovascular outcomes, which can yield greater cardiovascular benefits compared with the treatment strategy based on a single risk factor [7-10]. Statins are used as common lipid-lowering drugs globally for preventing CVD [11]. Recently, a synergistic effect of combined statin and antihypertensive therapy contributed to the prevention of CVD progression [12]. However, evidence supporting the use of statins combined with blood pressure-lowering medications for treating patients with grade 1 hypertension, irrespective of cholesterol levels, is lacking [13].

Patients with hypertension present in the moderate-risk category should be treated with statins [14]. Moreover, lifestyle intervention should be implemented to improve blood pressure levels before using antihypertensive and statin treatments [15]. However, studies reported inconsistent results regarding the effect of combined statin and antihypertensive therapy on major cardiovascular outcomes [16-23]. This might be because recruited patients did not "purely" have hypertension, which always combined with other cardiovascular risk factors. In a previous meta-analysis, combined statins and intense blood pressure-lowering regimen were compared in terms of their effects on major cardiovascular outcomes [12], but the study did not explore the effect of adding statins to antihypertensive therapy for treating patients with hypertension compared with antihypertensive therapy alone. Therefore, this systematic review and meta-analysis based on the available randomized controlled trials (RCTs) was conducted to evaluate and compare the effects of combined statin and antihypertensive therapy and antihypertensive therapy alone based on the patients' characteristics.

\section{Methods}

\section{Registration and Trial Identification}

The study protocol has been registered in the international prospective register of systematic reviews (PROSPERO), with the registration number CRD42017071935 [24]. This meta-analysis was conducted according to the Preferred Reporting Items for Systematic Reviews and Meta-Analyses guidelines [25].
Literature Searches and Study Selection

PubMed, Embase, and the Cochrane Library were searched from inception till October 9, 2019, without any limitations to language or publication status (in press or published). Studies with randomized controlled design and those investigating the treatment effects of combined statin and antihypertensive therapy on major adverse cardiovascular events (MACEs) were eligible for inclusion in the present meta-analysis. The core search terms were "statin" AND "antihypertensive drugs" AND "hypertension" AND "randomized controlled trials." The search strategy used in PubMed is presented in online supplement 1 (for all online suppl. material, see www.karger.com/doi/10.1159/000508280). The reference lists of all relevant RCTs were also checked for identifying any new eligible studies.

Two authors screened the search results independently for the inclusion of eligible RCTs, and any disagreements between them were resolved by reaching a consensus. The corresponding author made the final decision. The study was eligible for inclusion if the following criteria were met: (1) patients: patients with hypertension, irrespective of other cardiovascular risk factors; (2) intervention: patients received statins plus antihypertensive drugs; (3) control: patients received antihypertensive drugs alone; (4) outcomes: the study reported at least one of the following outcomes: MACEs (the definition of MACEs in individual trials are presented in online suppl. 2), myocardial infarction (MI), stroke, cardiac death, and all-cause mortality; and (5) study design: studies designed as RCTs. Studies with an observational design were excluded because the uncontrolled factors could bias the results.

\section{Data Extraction and Quality Assessment}

Two authors extracted the data independently regarding the identification of the study, methods of study design, information pertaining to participants and interventions, and outcomes. The collected data included the first author's surname and study group name, publication year, country, sample size, disease status, mean age, number of men and women, risk stratification, comparison, intensity of statin therapy [26], body mass index (BMI), systolic blood pressure (SBP), diastolic blood pressure (DBP), percentage of smokers, percentage of diabetes mellitus (DM), percentage of coronary heart disease (CHD) history, reported outcomes, and follow-up durations. A third author cross-checked all the entries and arbitrated any discordance. If the information was lacking in any of the included studies, the study authors were contacted and requested for further information. However, these studies were eventually excluded if no reply was received from the authors. The Jadad scale was employed to assess the study quality, which is a comprehensive method for evaluating the quality of RCTs in a meta-analysis [27]. The Jadad scale was based on randomization, blinding, allocation concealment, withdrawals and dropouts, and use of intention-to-treat analysis, and the scoring system ranged from 0 to 5 . The study quality was evaluated by 2 authors, and the information was examined and adjudicated by an additional author by referring to the original studies.

\section{Statistical Analyses}

The effect of combined therapy and the risk of MACEs, MI, stroke, cardiac death, and all-cause mortality based on the events and the sample size in each group in individual trials was analysed. The random-effects model was employed to calculate the summary relative risks (RRs) and corresponding 95\% CIs to compare 


\begin{abstract}
Fig. 1. Literature search and selection.
the effects of combined therapy versus antihypertensive therapy alone on MACEs $[28,29]$. The heterogeneity among the included trials was evaluated using the $I^{2}$ and Q statistic, and a $p$ value $<0.10$ was regarded as significant heterogeneity [30, 31]. Sensitivity analyses for investigated outcomes were conducted by the sequential exclusion of each individual trial from the meta-analysis [32]. The univariate meta-regression [33] and subgroup analyses were conducted for all reported outcomes based on sample size, disease status, mean age, intensity of statin therapy, and follow-up duration. Moreover, the subgroup analyses for MACEs based on additional characteristics (BMI, SBP, DBP, percentage of smokers, percentage of DM, and percentage of CHD history) were also performed. The potential publication biases for MACEs, MI, stroke, cardiac death, and all-cause mortality were calculated using the Begg's test $[34,35]$. The significant levels were 2 sided, and $p$ values $<0.05$ were considered to be significant for pooled outcomes. All statistical analyses were conducted using Stata software version 10.0 (Stata Corporation, TX, USA).
\end{abstract}

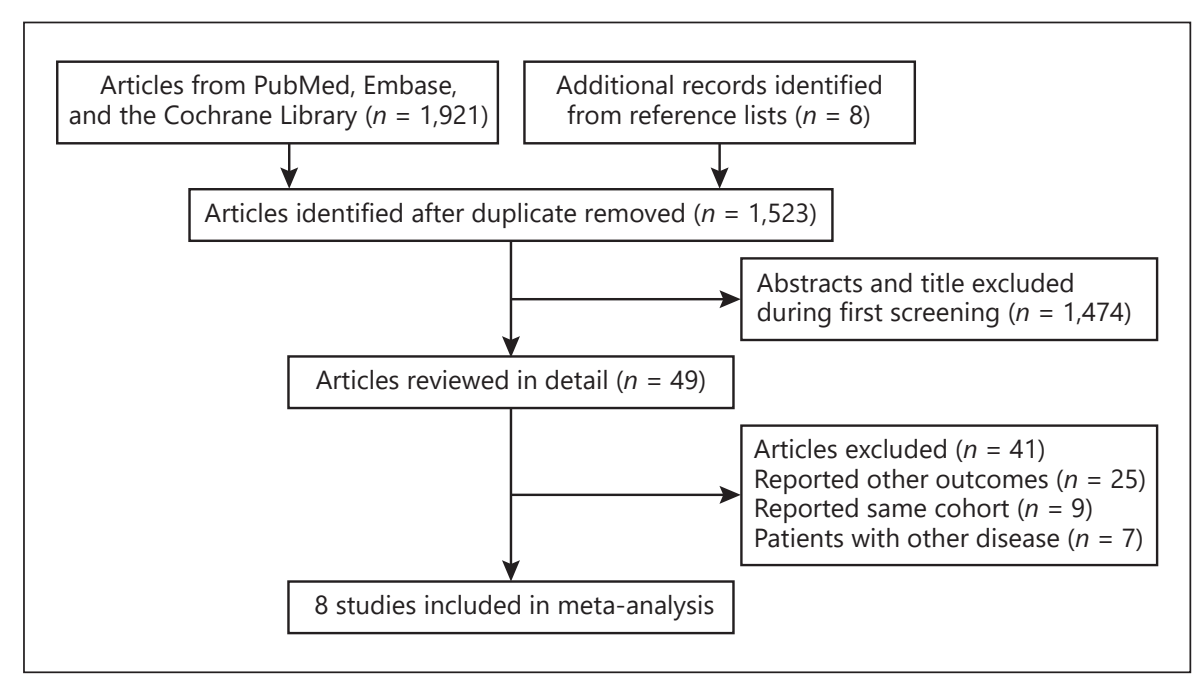

\section{Results}

The present study investigated and compared the effects of combined statin and antihypertensive therapy with those of antihypertensive therapy alone on major cardiovascular outcomes; the details of comparisons have been described in the protocol [24]. The initial electronic search generated 1,921 records; 1,872 studies were excluded due to duplications and irrelevant topics. A total of 49 potentially eligible trials were selected, and 41 were excluded because of the following reasons: study reporting other outcomes $(n=25)$, study reporting the same cohort $(n=9)$, and patients diagnosed with other diseases $(n=7)$. Finally, 8 RCTs were selected for this meta- analysis after detailed evaluations [16-23]. The manual searching of the reference lists of eligible studies yielded no additional trials. The results of the study selection process are presented in Figure 1, and the general baseline characteristics of trials and patients are shown in Table 1 and online supplement 2 .

Eight RCTs including a total of 38,618 patients with hypertension were enrolled in the final meta-analysis. The follow-up duration for patients was 3.0-6.0 years, while 396-10,355 patients were included in each trial. Three trials included patients with simple hypertension, while the remaining 5 trials included the concomitant occurrence of hypertension due to other diseases. Furthermore, 3 trials included patients receiving a low intensity of statins, and the remaining 5 trials included patients receiving a moderate intensity of statins. Five studies were conducted in Western countries, one in Asia, and the remaining 2 studies in multiple countries. The study quality was evaluated using the Jadad scale; all the studies included in this meta-analysis were of high quality because 3 trials scored 5 and 5 trials scored 4 (these trials did not report allocation concealment, introducing a potential risk of bias; Tables 1,2).

Eight trials reported the effect of combined therapy on MACEs, which included 38,618 patients with hypertension and recorded 2,548 MACEs. Combined therapy demonstrated a $21 \%$ reduction in the risk of MACEs compared with antihypertensive therapy alone (RR $0.79 ; 95 \%$ CI $0.71-0.88 ; p<0.001$; Fig. 2 ). Moderate heterogeneity was detected across the included trials $\left(I^{2}: 35.9 \% ; p=0.142\right)$, and the conclusion remained unchanged after the sequential exclusion of each individual trial (online suppl. 3). 


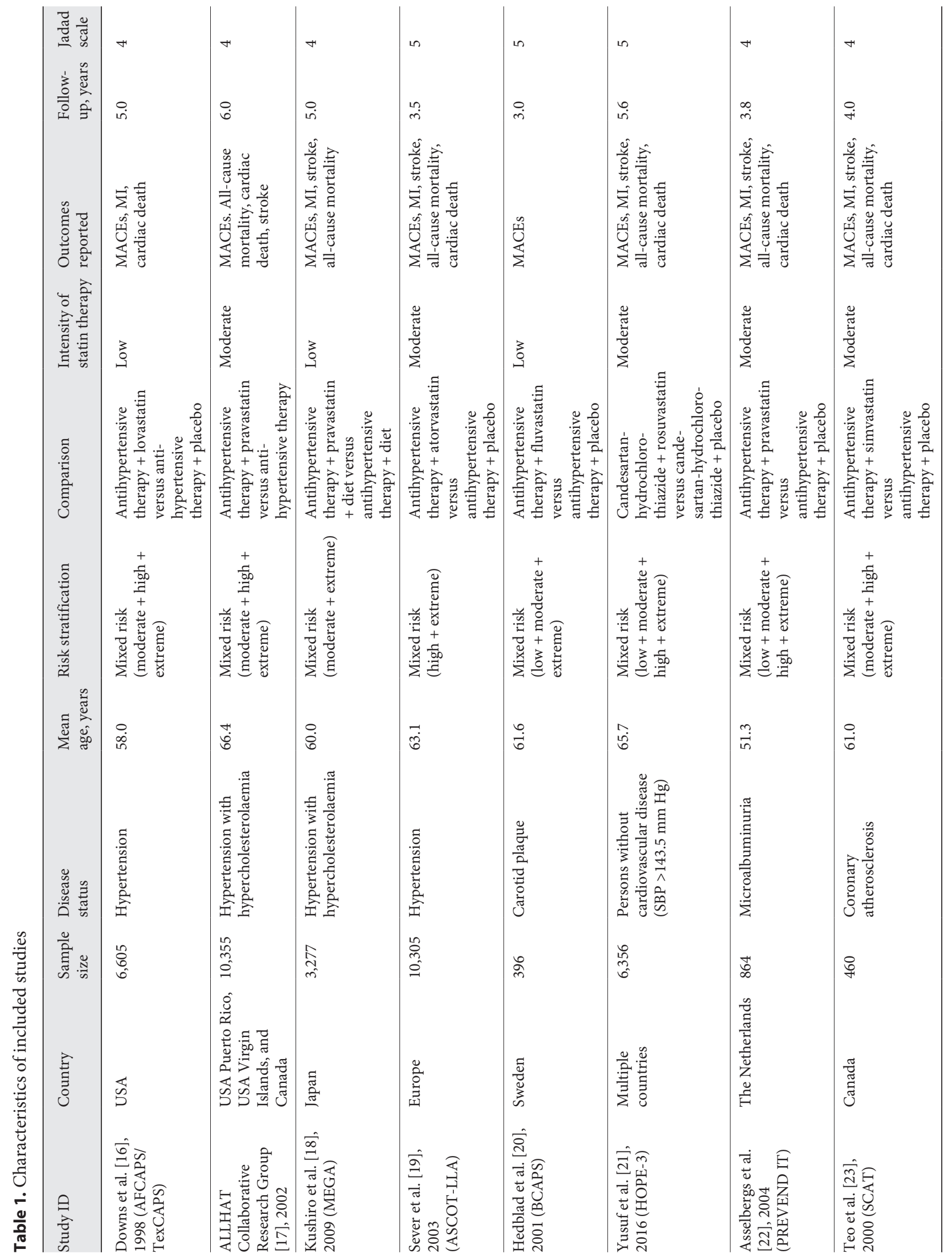




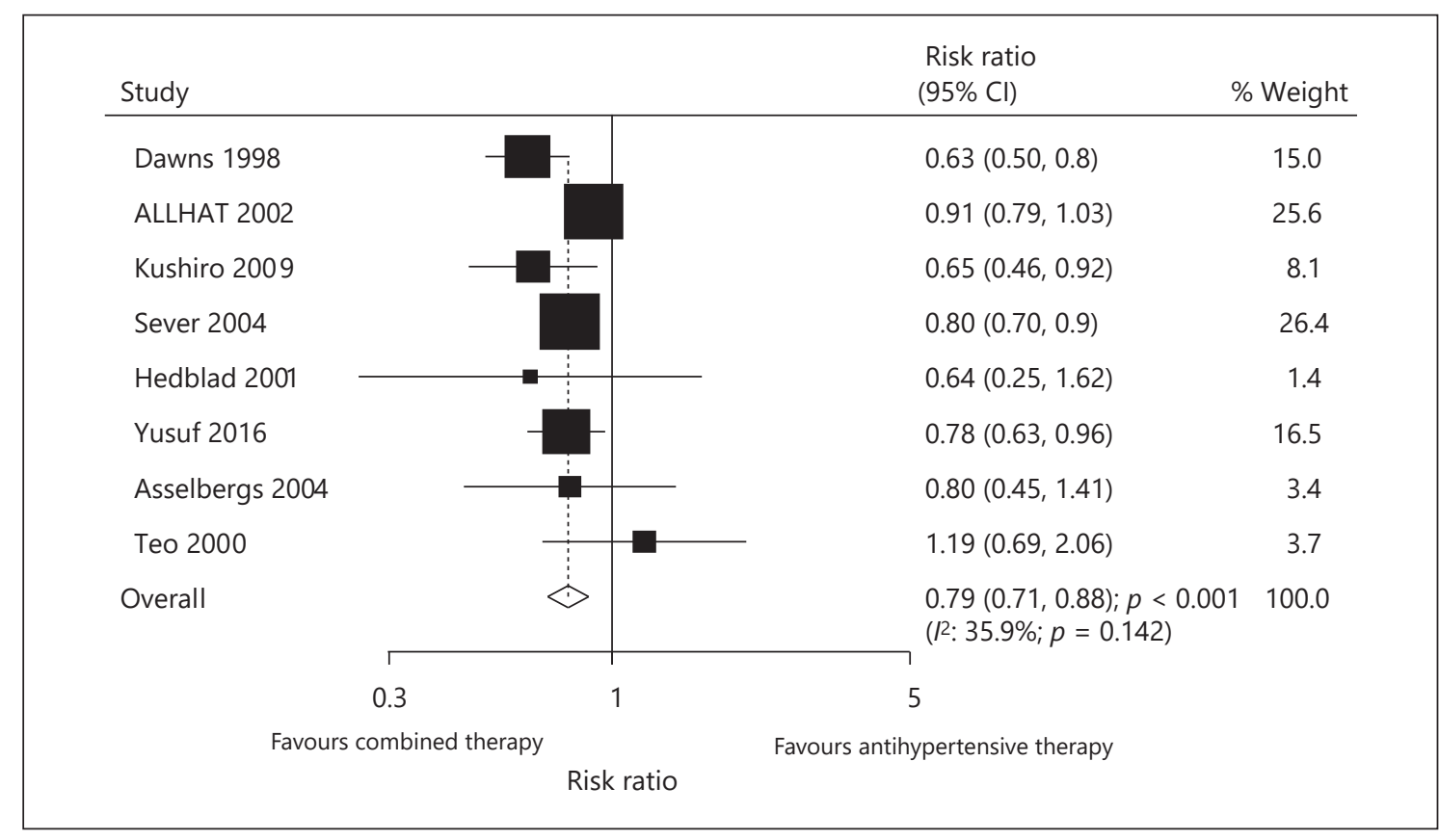

Fig. 2. Effect of combined therapy on major cardiovascular outcomes.

Table 2. The quality assessment of included studies

\begin{tabular}{|c|c|c|c|c|c|c|}
\hline Variable & Randomization & $\begin{array}{l}\text { Allocation } \\
\text { concealment }\end{array}$ & Blinding & $\begin{array}{l}\text { Lost to } \\
\text { follow-up }\end{array}$ & $\begin{array}{l}\text { Use of } \\
\text { ITT analysis }\end{array}$ & Overall \\
\hline Downs et al. [16], 1998 (AFCAPS/TexCAPS) & Yes & NA & Yes & Yes & Yes & Low risk \\
\hline Kushiro et al. [18], 2009 (MEGA) & Yes & NA & Yes & Yes & Yes & Low risk \\
\hline Sever et al. [19], 2003 (ASCOT-LLA) & Yes & Yes & Yes & Yes & Yes & Low risk \\
\hline Hedblad et al. [20], 2001 (BCAPS) & Yes & Yes & Yes & Yes & Yes & Low risk \\
\hline Asselbergs et al. [22], 2004 (PREVEND IT) & Yes & NA & Yes & Yes & Yes & Low risk \\
\hline Teo et al. [23], 2000 (SCAT) & Yes & NA & Yes & Yes & Yes & Low risk \\
\hline
\end{tabular}

Six trials reported the effect of combined therapy on MI, which included 27,867 patients with hypertension and recorded $307 \mathrm{MI}$ events. Overall, combined therapy reduced the risk of MI by $33 \%$ compared with antihypertensive therapy alone (RR 0.67 ; 95\% CI $0.53-0.84$; $p=0.001$; Fig. 3). Although no evidence of heterogeneity among the included trials was observed $\left(I^{2}: 0.0 \%\right.$; $p=0.770$ ), the results showed no significant difference after excluding the study conducted by Downs et al. [16]. The trial specifically reported a high incidence of MI and patients were on a low-saturated and low-cholesterol diet (online suppl. 3). When excluding the trial conducted by Downs et al. [16], the power was not enough to detect a potential difference between the ef- fects of combined therapy and those of antihypertensive therapy alone.

Six trials reported the effect of combined therapy on stroke, which included 31,617 patients with hypertension and recorded 815 stroke events. Overall, combined therapy reduced the risk of stroke by $18 \%$ compared with antihypertensive therapy alone (RR 0.82; 95\% CI 0.72-0.94; $p=0.005$; Fig. 4). Although no significant heterogeneity among the included trials was observed $\left(I^{2}: 0.0 \% ; p=\right.$ 0.456 ), the sensitivity analysis was conducted by sequentially excluding each trial. The result suggested no significant difference between combined therapy and antihypertensive therapy alone with regard to stroke (online suppl. 3) when excluding the study conducted by Sever et 


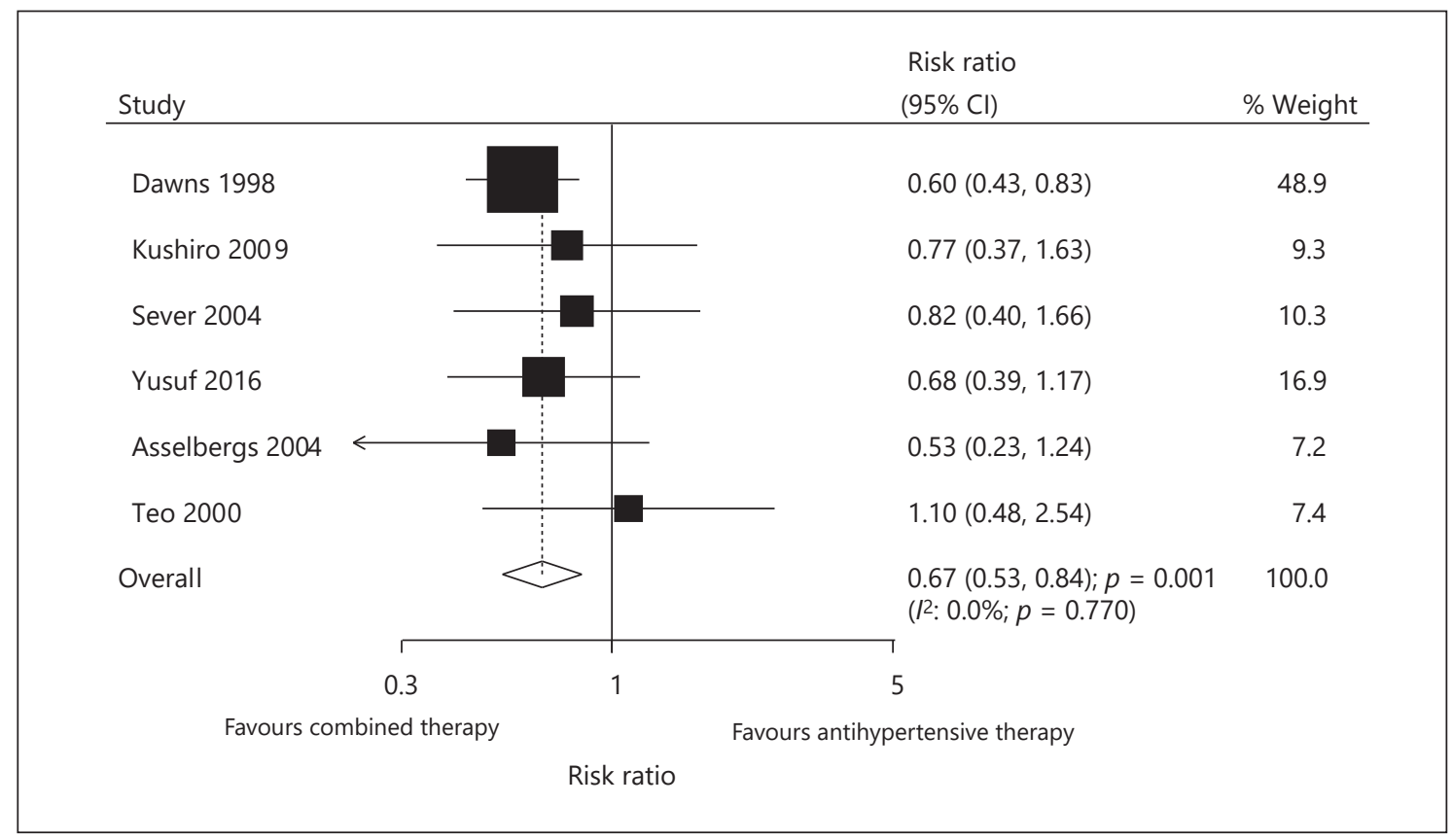

Fig. 3. Effect of combined therapy on myocardial infarction.

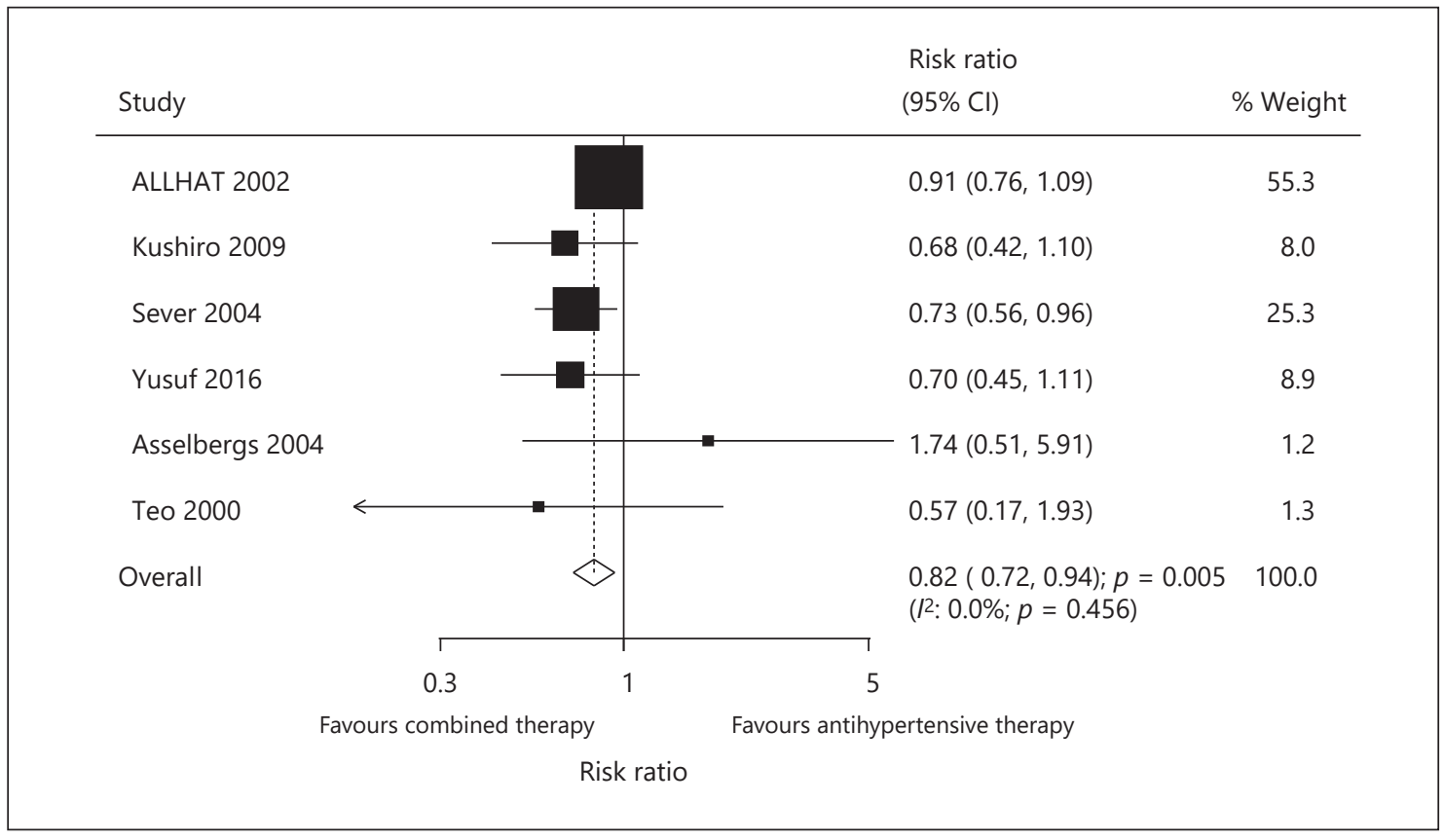

Fig. 4. Effect of combined therapy on stroke.

al. [19]. This was probably because the trial conducted by Sever et al. [19] included patients at high risk of stroke, and the treatment effects were greater than those on patients at low risk.

Combined Medication for Hypertension
Six trials reported the effect of combined therapy on cardiac death, which included 34,945 patients with hypertension and recorded 967 cardiac deaths. Overall, no study showed that combined therapy could reduce the 


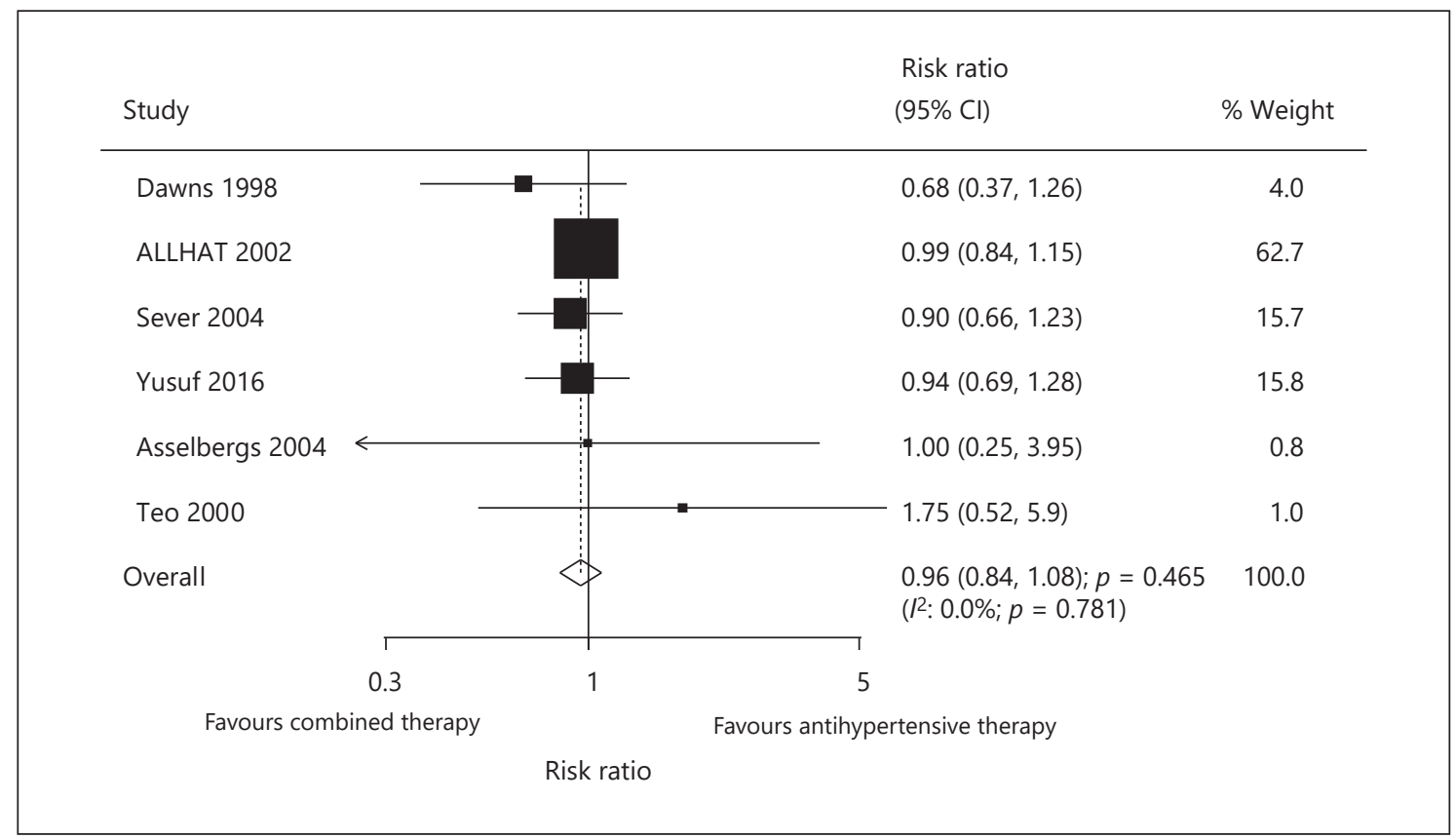

Fig. 5. Effect of combined therapy on cardiac death.

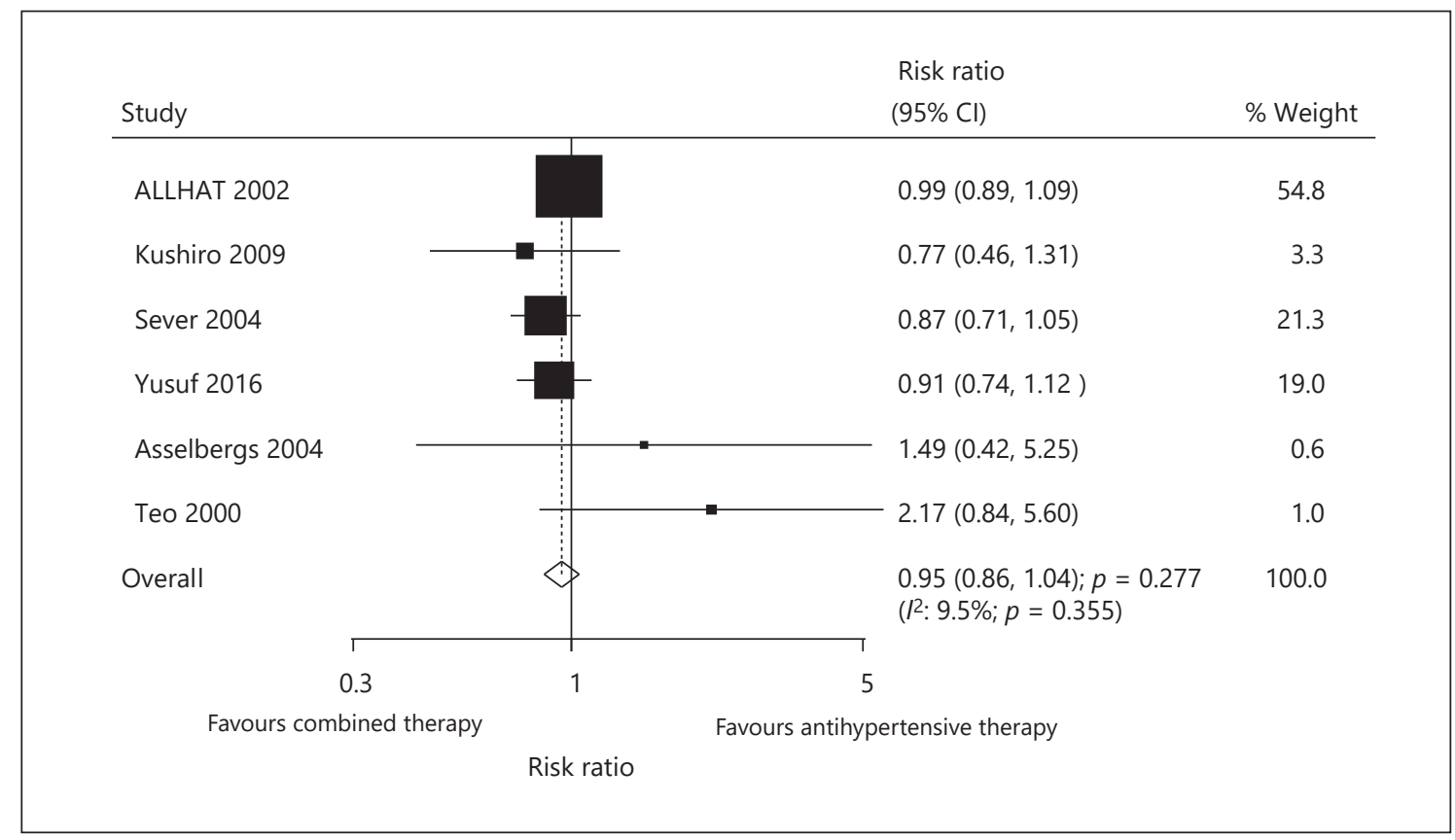

Fig. 6. Effect of combined therapy on all-cause mortality.

risk of cardiac death (RR 0.96; 95\% CI 0.84-1.08; $p=$ 0.465 ; Fig. 5). Further, no evidence of heterogeneity among the included trials $\left(I^{2}: 0.0 \% ; p=0.781\right)$ was found after each study was sequentially excluded from the pooled analysis. The conclusion was not affected by the exclusion of any specific study (online suppl. 3).

Six trials reported the effect of combined therapy on all-cause mortality, which included 31,617 patients with 
Table 3. Subgroup analysis for MACEs

\begin{tabular}{|c|c|c|c|c|c|c|}
\hline Variable group & Trials, $n$ & RR (95\% CI) & $p$ value & $I^{2}, \%$ & $\begin{array}{l}p \text { value for } \\
\text { heterogeneity }\end{array}$ & $\begin{array}{l}p \text { value for } \\
\text { meta-regression }\end{array}$ \\
\hline \multicolumn{7}{|l|}{ Sample size } \\
\hline$\geq 1,000$ & 5 & $0.78(0.68-0.88)$ & $<0.001$ & 54.2 & 0.068 & 0.464 \\
\hline$<1,000$ & 3 & $0.92(0.64-1.32)$ & 0.650 & 0.0 & 0.436 & \\
\hline \multicolumn{7}{|l|}{ Disease status } \\
\hline \multicolumn{7}{|l|}{ Mean age } \\
\hline$\geq 65.0$ years & 2 & $0.86(0.75-0.99)$ & 0.032 & 26.2 & 0.245 & 0.074 \\
\hline$<65.0$ years & 6 & $0.75(0.65-0.86)$ & $<0.001$ & 21.8 & 0.270 & \\
\hline \multicolumn{7}{|l|}{ Intensity of statin therapy } \\
\hline Low & 3 & $0.64(0.53-0.77)$ & $<0.001$ & 0.0 & 0.990 & 0.008 \\
\hline Moderate & 5 & $0.84(0.77-0.91)$ & $<0.001$ & 0.0 & 0.414 & \\
\hline
\end{tabular}

hypertension and recorded 2,096 all-cause mortalities. The combined therapy had no significant effect on the risk of all-cause mortality compared with antihypertensive therapy alone (RR $0.95 ; 95 \%$ CI $0.86-1.04 ; p=0.277$; Fig. 6). An unimportant heterogeneity was noted among the included trials, and the results of sensitivity analyses showed no significant differences after excluding any individual trial (online suppl. 3).

Although moderate or unimportant heterogeneity was detected for the investigated outcomes, univariate meta-regression and subgroup analyses were conducted to explore potential sources of heterogeneity. The treatment effects of combined therapy versus antihypertensive therapy alone on MACEs were affected by disease status, mean age of included patients, and intensity of statin therapy. Although significant differences were observed in most of the subsets, the combined therapy showed no association with the risk of MACEs if the sample size of the trials was $<1,000$ or patients also had other diseases (Table 3). Moreover, subgroup analyses based on additional patient characteristics were also conducted, and most of the results were consistent with the overall analysis results. However, the combined therapy did not yield additional benefits against the risk of MACE if the history of $\mathrm{CHD} \geq 10 \%$ (online suppl. 4). Furthermore, the subgroup analyses indicated that combined therapy significantly reduced the risk of $M I$ if the sample size of the trials was $\geq 1,000$, patients had simple hypertension, the mean age of patients was $<65.0$ years, patients received a low intensity of statins, and the duration of follow-up was $\geq 5.0$ years (online suppl. 5). Moreover, the risk of stroke was significantly reduced in patients who received combined therapy if the sample size of the trials was $\geq 1,000$, patients had simple hypertension, the mean age of patients was $<65.0$ years, patients received a moderate intensity of statins, and the duration of followup was $\geq 5.0$ years (online suppl. 5). Finally, no significant differences were observed between combined therapy and antihypertensive therapy alone with regard to cardiac death and all-cause mortality in all the subsets (online suppl. 5).

Publication biases for investigated outcomes were assessed using the Begg test [34]. No significant publication biases for MACEs $(p=0.711)$, MI $(p=0.452)$, stroke ( $p=$ $0.707)$, cardiac death $(p=1.000)$, and all-cause mortality $(p=0.707)$ were observed.

\section{Discussion}

This systematic review and meta-analysis directly compared the effects of combined therapy versus antihypertensive therapy alone on major cardiovascular outcomes in patients with hypertension. It included a total of 8 RCTs with 38,618 patients having a wide range of characteristics. The summary results indicated that combined therapy significantly reduced the risk of MACEs, MI, and stroke, but had no significant effect on cardiac death and all-cause mortality compared with antihypertensive therapy alone. Further, subgroup analyses suggested that combined therapies for patients with simple hypertension, with a mean age $<65.0$ years, receiving a low inten- 
sity of statins, BMI $<28.0 \mathrm{~kg} / \mathrm{m}^{2}$, and percentage of smokers $<20.0 \%$ offered greater benefits against MACEs compared with corresponding subsets.

A meta-analysis conducted by Briasoulis et al. [36] included 40 studies and 45,113 patients. The results showed that patients taking statins contributed to a small but statistically significant reduction of SBP and DBP. Another important meta-analysis suggested that statins greatly reduced blood pressure unrelated to age, changes in serum cholesterol levels, and length of the trial [37]. However, these studies demonstrated the effectiveness of statins on blood pressure, but the clinical endpoints after long-term statin therapy were not investigated. Messerli et al. [38] conducted a meta-analysis of 12 RCTs, and pooled estimates of RR for patients with and without hypertension were calculated separately. The results showed that patients with and without hypertension receiving statin therapy had reduced cardiovascular morbidity and mortality. Furthermore, Sundström et al. [12] suggested that blood pressure- lowering drugs and statins provided multiple benefits in terms of cardiovascular outcomes. However, the direct comparison of major cardiovascular outcomes of combined therapy versus antihypertensive drugs alone was not performed. Therefore, this quantitative meta-analysis of RCTs was conducted to evaluate the treatment effects of combined therapy versus antihypertensive drugs alone on major cardiovascular outcomes in patients with hypertension.

The results showed that the addition of statins in hypertensive patients was more beneficial against MACEs. However, several studies included in the present metaanalysis reported inconsistent results. The ALLHAT Collaborative Research Group conducted a trial including 10,355 moderately ambulatory patients with hypercholesterolemia and patients with hypertension, and suggested that pravastatin did not provide additional benefits against MACEs, stroke, cardiac death, or all-cause mortality compared with usual care. This was probably because of the imbalances in total cholesterol and low-density lipoprotein cholesterol levels between the pravastatin and usualcare groups [17]. Furthermore, nearly half of the patients had high risk, including $15 \%$ of patients with a CVD history and $35 \%$ of patients with type 2 diabetes, and the mean age of patients was $>66.0$ years, suggesting that statins might offer a greater benefit in the primary prevention of MACEs. Moreover, Hedblad et al. [20] found that the number of patients with MACEs in the combined therapy and antihypertensive therapy alone groups was 7 and 11, respectively. Asselbergs et al. [22] showed that adding pravastatin did not have an additional effect on urinary albumin excretion and MACEs in patients with hypertension and microalbuminuria. Teo et al. [23] indicated that fewer patients receiving enalapril experienced the combined endpoint of death, MI, and stroke compared with those who received simvastatin plus enalapril therapies ( 25 vs. 21 ). This was probably because the smaller sample size and the shorter duration of follow-up showed a clinical benefit, especially if event rates were lower than expected. This easily led to broader 95\% CIs, resulting in no statistically significant differences. Moreover, the use of combined therapy offered significant benefits against MACEs because of the comorbidity and history of other macrovascular diseases in patients who received statins to reduce the traditional risk factors.

The risk of MI and stroke significantly decreased in the combined therapy group, while no significant difference in the incidence of cardiac death and all-cause mortality was observed. Significant differences in the incidence of MI and stroke were shown mainly in the trials conducted by Downs et al. [16] and Sever et al. [19], respectively. The cardiac death and all-cause mortality could be affected by the trial conducted by the ALLHAT Collaborative Research Group [17], and hence no significant differences were observed. These trials contributed to high weighted effect estimates from the overall analysis. Several other reasons contributed to the interpretation of these results: (1) patients in the included trials demonstrated varied cardiovascular risk stratifications, affecting the progression of MACEs; (2) patients in 5 of 8 trials also had other diseases, playing an important role in the risk of MACEs; and (3) the statin types and intensity were different and offered varied benefits against MACEs [39, 40].

The subgroup analysis suggested that combined therapy provided greater benefits against MACEs if the sample size of the included trials was $\geq 1,000$, patients had simple hypertension, the mean age of included patients was $<65.0$ years, patients received a low intensity of statins, and trials had longer follow-up durations. Similar results were observed for MI and stroke, while a moderate intensity of statins provided more benefits against stroke. First, the sample size correlated with statistical power and contributed to high weighted effect estimates from the pooled analysis. Second, the combined therapy demonstrated greater benefits for the primary prevention of MACEs, and the potential risk factors could be adjusted using combined statin and hypertensive therapy. Third, the mean age of patients $<65.0$ years was associated with greater benefits due to low cardiovascular risk and high drug absorption capacity. It is interesting because younger patients are always associated with a lower percentage 
of comorbidities. Also, the treatment effects of combined therapies on MACEs in younger patients may be due to the addition of statins to antihypertensive therapies, which has a synergistic effect on blood pressure. Fourth, a low intensity of statins is always used for primary prevention, yielding more benefits. Fifth, the duration of follow-up could affect the prevalence of MACEs in individual trials and the weighted effect estimates in the pooled results. Moreover, the risk of stroke with a greater reduction in patients who received a moderate dose of statins was determined by the trial conducted by Sever et al. [19]. Therefore, these conclusions might vary, and the relative results were provided in the current meta-analysis. Finally, most of the results of the subgroup analyses based on BMI, SBP, DBP, percentage of smokers, percentage of $\mathrm{DM}$, and percentage of CHD history indicated that combined therapy was superior to antihypertensive drugs alone, whereas no significant differences were observed in the risk of MACEs when the history of CHD was $\geq 10 \%$.

This meta-analysis had several advantages, such as the use of professional search strategies and strict inclusion and exclusion criteria. However, it also had several limitations. First, different antihypertensives and statins used in the included studies might affect the risk of cardiovascular outcomes. However, stratified analyses based on these factors were not performed due to the smaller number of included trials. Second, the included trials involved patients with different cardiovascular risks, and all the studies were defined as "mixed risk." Consequently, a more detailed analysis could not be performed. Third, the definition for MACEs varied across the included trials, affecting the pooled results. Fourth, whether the beneficial effects of combined therapies originated from the blood pressure-lowering effect was not illustrated. This was because the data regarding the changes in blood pressure were not available in all the included trials. Finally, this meta-analysis was based on published studies and used pooled data; hence, the inherent limitations of publication bias and restricted analyses were inevitable.
In conclusion, the present meta-analysis indicated that combined therapy offered greater benefits against MACEs, MI, and stroke compared with antihypertensive drugs alone. However, no significant differences were found between these 2 interventions in terms of the risk of cardiac death and all-cause mortality. Furthermore, large-scale RCTs focusing on the intensity and type of statins in patients with hypertension for the primary prevention of MACEs should be conducted to verify these findings.

\section{Statement of Ethics}

This meta-analysis was conducted according to the Preferred Reporting Items for Systematic Reviews and Meta-Analyses guidelines. The study protocol was registered in the international prospective register of systematic reviews (PROSPERO) (registration No. CRD42017071935). All analyses were based on previously published studies, and hence no ethical approval and patient consent were required.

\section{Disclosure Statement}

The authors declare no conflicts of interest.

\section{Funding Sources}

This study was supported by the Foundation of Beijing Anzhen Hospital, Capital Medical University (2016P01), and the Clinical Vascular Grant in Chinese Physicians (2017-CCA-VG-016).

\section{Author Contributions}

Y.W. and L.J. performed the experiments and wrote the manuscript. Y.W., L.J., S.-J.F., and X.-Y.T. carried out the data collection and analysis. Z.-M.K. conceived and coordinated the study, and revised the manuscript. All authors reviewed the results and approved the final version of the manuscript.

\section{References}

1 Kannel WB. Risk stratification in hypertension: new insights from the Framingham Study. Am J Hypertens. 2000 Jan;13(1 Pt 2): 3S-10S.

2 Ettehad D, Emdin CA, Kiran A, Anderson SG, Callender T, Emberson J, et al. Blood pressure lowering for prevention of cardiovascular disease and death: a systematic review and metaanalysis. Lancet. 2016 Mar;387(10022):95767.
3 Krogsbøll LT, Jørgensen KJ, Grønhøj Larsen C, Gøtzsche PC. General health checks in adults for reducing morbidity and mortality from disease. Cochrane Database Syst Rev. 2012 Oct; 10:CD009009.

4 Giles TD, Materson BJ, Cohn JN, Kostis JB. Definition and classification of hypertension: an update. J Clin Hypertens (Greenwich). 2009 Nov;11(11):611-4.
5 Cuspidi C, Sala C, Tadic M, Rescaldani M, De Giorgi GA, Grassi G, et al. Untreated masked hypertension and carotid atherosclerosis: a meta-analysis. Blood Press. 2015 Apr;24(2):65-71.

6 Miller JD, Aronis KN, Chrispin J, Patil KD, Marine JE, Martin SS, et al. Obesity, Exercise, Obstructive Sleep Apnea, and Modifiable Atherosclerotic Cardiovascular Disease Risk Factors in Atrial Fibrillation. J Am Coll Cardiol. 2015 Dec;66(25):2899-906. 
7 Wald NJ, Law MR. A strategy to reduce cardiovascular disease by more than $80 \%$. BMJ. 2003 Jun;326(7404):1419.

8 Sundström J, Neal B. Replacing the hypertension control paradigm with a strategy of cardiovascular risk reduction. Eur Heart J Qual Care Clin Outcomes. 2015 Jul;1(1):17-22.

9 Cholesterol Treatment Trialists' (CTT) Collaborators. Mihaylova B, Emberson J, Blackwell L, Keech A, Simes J, Barnes EH, et al. The effects of lowering LDL cholesterol with statin therapy in people at low risk of vascular disease: meta-analysis of individual data from 27 randomised trials. Lancet. 2012 Aug; 380(9841):581-90.

10 Blood Pressure Lowering Treatment Trialists Collaboration. Blood pressure-lowering treatment based on cardiovascular risk: a meta-analysis of individual patient data. Lancet. 2014 Aug;384(9943):591-8.

11 Cholesterol Treatment Trialists' (CTT) Collaborators. Kearney PM, Blackwell L, Collins R, Keech A, Simes J, Peto R, et al. Efficacy of cholesterol-lowering therapy in 18,686 people with diabetes in 14 randomised trials of statins: a meta-analysis. Lancet. 2008 Jan; 371(9607):117-25.

12 Sundström J, Gulliksson G, Wirén M. Synergistic effects of blood pressure-lowering drugs and statins: systematic review and meta-analysis. BMJ Evid Based Med. 2018 Apr;23(2): 64-9.

13 Mancia G, Parati G, Revera M, Bilo G, Giuliano A, Veglia F, et al. Statins, antihypertensive treatment, and blood pressure control in clinic and over 24 hours: evidence from PHYLLIS randomised double blind trial. BMJ. 2010 Mar;340:c1197.

14 Mortensen MB, Budoff M, Li D, Nasir K, Blaha MJ, Sandfort V, et al. High-Quality Statin Trials Support the 2013 American College of Cardiology/American Heart Association Cholesterol Guidelines After the HOPE-3 Trial (Heart Outcomes Prevention Evaluation-3): MESA (The Multiethnic Study of Atherosclerosis). Circulation. 2017 Nov; 136(19):1863-5.

15 Hjelstuen A, Anderssen SA, Holme I, Seljeflot I, Klemsdal TO. Effect of lifestyle and/or statin treatment on soluble markers of atherosclerosis in hypertensives. Scand Cardiovasc J. 2007 Oct;41(5):313-20.

16 Downs JR, Clearfield M, Weis S, Whitney E, Shapiro DR, Beere PA, et al.; for the AFCAPS/ TexCAPS Research Group. Primary prevention of acute coronary events with lovastatin in men and women with average cholesterol levels: results of AFCAPS/TexCAPS. Air Force/ Texas Coronary Atherosclerosis Prevention Study. JAMA. 1998 May;279(20):1615-22.

17 ALLHAT Officers and Coordinators for the ALLHAT Collaborative Research Group. The Antihypertensive and Lipid-Lowering Treatment to Prevent Heart Attack Trial. Major outcomes in moderately hypercholesterolemic, hypertensive patients randomized to pravastatin vs usual care: The Antihypertensive and Lipid-Lowering Treatment to Prevent Heart Attack Trial (ALLHAT-LLT). JAMA. 2002 Dec;288(23):2998-3007.

18 Kushiro T, Mizuno K, Nakaya N, Ohashi Y, Tajima N, Teramoto T, et al.; Management of Elevated Cholesterol in the Primary Prevention Group of Adult Japanese Study Group. Pravastatin for cardiovascular event primary prevention in patients with mild-to-moderate hypertension in the Management of Elevated Cholesterol in the Primary Prevention Group of Adult Japanese (MEGA) Study. Hypertension. 2009 Feb;53(2):135-41.

19 Sever PS, Dahlöf B, Poulter NR, Wedel H, Beevers G, Caulfield M, et al.; ASCOT investigators. Prevention of coronary and stroke events with atorvastatin in hypertensive patients who have average or lower-than-average cholesterol concentrations, in the AngloScandinavian Cardiac Outcomes Trial-Lipid Lowering Arm (ASCOT-LLA): a multicentre randomised controlled trial. Lancet. 2003 Apr;361(9364):1149-58.

20 Hedblad B, Wikstrand J, Janzon L, Wedel H, Berglund G. Low-dose metoprolol CR/XL and fluvastatin slow progression of carotid intima-media thickness: Main results from the Beta-Blocker Cholesterol-Lowering Asymptomatic Plaque Study (BCAPS). Circulation. 2001 Apr;103(13):1721-6.

21 Yusuf S, Lonn E, Pais P, Bosch J, López-Jaramillo P, Zhu J, et al.; HOPE-3 Investigators. Blood-Pressure and Cholesterol Lowering in Persons without Cardiovascular Disease. N Engl J Med. 2016 May;374(21):2032-43.

22 Asselbergs FW, Diercks GF, Hillege HL, van Boven AJ, Janssen WM, Voors AA, et al.; Prevention of Renal and Vascular Endstage Disease Intervention Trial (PREVEND IT) Investigators. Effects of fosinopril and pravastatin on cardiovascular events in subjects with microalbuminuria. Circulation. 2004 Nov; 110(18):2809-16.

23 Teo KK, Burton JR, Buller CE, Plante S, Catellier D, Tymchak W, et al. Long-term effects of cholesterol lowering and angiotensin-converting enzyme inhibition on coronary atherosclerosis: The Simvastatin/Enalapril Coronary Atherosclerosis Trial (SCAT). Circulation. 2000 Oct;102(15):1748-54.

24 Wang Y, Kuang ZM, Feng SJ, Jiang L, Chen QX, Ji XY, et al. Combined antihypertensive and statin therapy for the prevention of cardiovascular events in patients with hypertension without complications: protocol for a systematic review and meta-analysis. BMJ Open. 2018 May;8(5):e019719.

25 Moher D, Liberati A, Tetzlaff J, Altman DG; PRISMA Group. Preferred reporting items for systematic reviews and meta-analyses: the PRISMA statement. PLoS Med. 2009 Jul; 6(7):e1000097.

26 Arnold SV, Kosiborod M, Tang F, Zhao Z, Maddox TM, McCollam PL, et al. Patterns of statin initiation, intensification, and maximi- zation among patients hospitalized with an acute myocardial infarction. Circulation. 2014 Mar;129(12):1303-9.

27 Jadad AR, Moore RA, Carroll D, Jenkinson C, Reynolds DJ, Gavaghan DJ, et al. Assessing the quality of reports of randomized clinical trials: is blinding necessary? Control Clin Trials. 1996 Feb;17(1):1-12.

28 DerSimonian R, Laird N. Meta-analysis in clinical trials. Control Clin Trials. 1986 Sep; 7(3):177-88.

29 Ades AE, Lu G, Higgins JP. The interpretation of random-effects meta-analysis in decision models. Med Decis Making. 2005 Nov-Dec; 25(6):646-54.

30 Deeks J, Higgins J, Altman D. Analyzing data and undertaking meta-analyses. In: Higgins J, Green S, editors. Cochrane Handbook for Systematic Reviews of Interventions. Version 5.0.1. Oxford: The Cochrane Collaboration; 2008.

31 Higgins JP, Thompson SG, Deeks JJ, Altman DG. Measuring inconsistency in meta-analyses. BMJ. 2003 Sep;327(7414):557-60.

32 Tobias A. Assessing the influence of a single study in meta-analysis. Stata Tech Bull. 1999; 47:15-7.

33 Thompson SG, Higgins JP. How should metaregression analyses be undertaken and interpreted? Stat Med. 2002 Jun;21(11):1559-73.

34 Begg CB, Mazumdar M. Operating characteristics of a rank correlation test for publication bias. Biometrics. 1994 Dec;50(4):1088-101.

35 Higgins J, Green S. 9.5 heterogeneity. In: Higgins J, Green S, editors. Cochrane handbook for systematic review of interventions 501 . Oxford, UK: The Cochrane Collabration; 2011.

36 Briasoulis A, Agarwal V, Valachis A, Messerli FH. Antihypertensive effects of statins: a meta-analysis of prospective controlled studies. J Clin Hypertens (Greenwich). 2013 May; 15(5):310-20

37 Strazzullo P, Kerry SM, Barbato A, Versiero M, D’Elia L, Cappuccio FP. Do statins reduce blood pressure?: a meta-analysis of randomized, controlled trials. Hypertension. 2007 Apr;49(4):792-8

38 Messerli FH, Pinto L, Tang SS, Thakker KM, Cappelleri JC, Sichrovsky T, et al. Impact of systemic hypertension on the cardiovascular benefits of statin therapy-a meta-analysis. Am J Cardiol. 2008 Feb;101(3):319-25.

39 Cholesterol Treatment Trialists' (CTT) Collaboration. Baigent C, Blackwell L, Emberson J, Holland LE, Reith C, Bhala N, et al. Efficacy and safety of more intensive lowering of LDL cholesterol: a meta-analysis of data from 170,000 participants in 26 randomised trials. Lancet. 2010 Nov;376(9753):1670-81

40 Mills EJ, O’Regan C, Eyawo O, Wu P, Mills F, Berwanger $\mathrm{O}$, et al. Intensive statin therapy compared with moderate dosing for prevention of cardiovascular events: a meta-analysis of $>40000$ patients. Eur Heart J. 2011 Jun; 32(11):1409-15. 